\title{
Coherent acoustic phonons in nanostructures investigated by asynchronous optical sampling
}

\author{
T. Dekorsy*a, F. Hudert ${ }^{\mathrm{a}}$, R. Cerna ${ }^{\mathrm{a}}$, H. Schäfer ${ }^{\mathrm{a}}$, C. Janke ${ }^{\mathrm{a}, \mathrm{b}}$, A. Bartels ${ }^{\mathrm{a}, \mathrm{b}}$, K. Köhler ${ }^{\mathrm{c}}$, S. Braun ${ }^{\mathrm{d}}$, \\ M. Wiemer ${ }^{\mathrm{e}}, \mathrm{S}$. Mantl ${ }^{\mathrm{f}}$ \\ ${ }^{a}$ Department of Physics and Center for Applied Photonics, University Konstanz, Box M700, 78457 \\ Konstanz, Germany \\ ${ }^{\mathrm{b}}$ Gigaoptics GmbH, Blarerstr. 56, D-78462, Konstanz, Germany \\ ${ }^{c}$ Fraunhofer-Institut für Angewandte Festkörperphysik, Tullastr. 72, 79108 Freiburg, \\ Germany \\ ${ }^{\mathrm{d}}$ Fraunhofer-Institut für Werkstoff- und Strahltechnik, Winterbergstr. 28, 01277 Dresden, Germany \\ ${ }^{\mathrm{e}}$ Fraunhofer-Institut für Zuverlässigkeit und Mikrointegration IZM, Reichenhainer Str. 88, 09126 \\ Chemnitz, Germany \\ fInstitut für Bio- und Nanosysteme, Forschungszentrum Juelich, 52425 Juelich, Germany
}

\begin{abstract}
A new optical pump-probe technique is implemented for the investigation of acoustic phonon dynamics in the $\mathrm{GHz}$ to $\mathrm{THz}$ frequency range which is based on two asynchronously linked femtosecond lasers. Asynchronous optical sampling (ASOPS) provides the performance of on all-optical oscilloscope and allows us to record optically induced lattice dynamics over nanosecond times with 200 femtoseconds resolution at scan rates of $10 \mathrm{kHz}$. The generation of coherent acoustic phonons and their propagation and decay dynamics are investigated in semiconductor heterostructures, layered nanoscale materials of relevance for microelectronics, and X-ray mirrors. Changes of the optical properties of tailored semiconductor heterostructures associated with coherent phonon dynamics open the pathway for the modulation of optical signals at up to $\mathrm{THz}$ frequencies.
\end{abstract}

Keywords: coherent phonons, asynchronous optical sampling, ASOPS, picosecond ultrasonics, GHz modulation, metrology, EUV mirrors, wafer bonding

\section{INTRODUCTION}

In the past decade, pump-probe experiments with ultrashort laser pulses have significantly contributed to our understanding of ultrafast fundamental interactions in semiconductors and semiconductor heterostructures [1]. Since the interplay of electron and lattice dynamics ultimately limits the performance of electronic and optoelectronic devices, these investigations provide essential information on the physical limits for high-speed optoelectronic modulation. Thermally populated phonons are usually considered to be the origin of scattering and energy dissipation for charge carriers in semiconductors and hence detrimental for high-frequency electronic dynamics. In contrast, the generation of coherent acoustic and optical phonons via femtosecond laser pulses opens the pathway to control lattice dynamics in amplitude and phase [2] and thus electrical and optical properties of semiconductor nanostructures can be modulated at phonon frequencies in a well defined way, e.g. via femtosecond pulse shaping methods for generating arbitrary optical waveforms [3]. We employ a new experimental technique based on two asynchronously linked femtosecond lasers with $\mathrm{GHz}$ repetition rate to study the dynamics of coherently generated acoustic phonons and phonon pulses in semiconductor heterostructures [4] and nanoscale multilayers with relevance in microelectronics.

*thomas.dekorsy@uni-konstanz.de; phone +49-7531-883820, fax +49-7531-883072, www.uni-konstanz.de/fuf/physik/dekorsy 
Folding of the Brillouin zone in semiconductor superlattices provides means for tailoring the phonon frequency at small wave-vectors from a few tens of $\mathrm{GHz}$ to the $\mathrm{THz}$ frequency range $[5,6]$. Such superlattices can be used to construct cavities for acoustic phonons. The combination of acoustic cavities with optical microcavities enables an enhancement of the interaction of sound waves with photons by orders of magnitude as has recently been shown in static Raman scattering experiments [7-9]. The prospects of using such high frequency phonons for the modulation of optical signals in photonic crystals or combined phonon-photon cavities are yet to be exploited. We show that the new optical pumpprobe technique of asynchronous optical sampling (ASOPS) is the most suited technique to foster developments in this field. In addition, we provide examples for the generation and detection of acoustic pulses in nanoscale layered materials such as Si-Mo multilayers for EUV mirrors or in waferbonded Si which demonstrates the benefits of ASOPS in the field of laser based ultrasonics and semiconductor metrology [10,11,12]. The paper is organized as follows: In Sec. 2 we introduce high-speed ASOPS with femtosecond lasers of GHz repetition rate as a key method for the detection of GHz to $\mathrm{THz}$ phonon dynamics. In Sec. 3 we describe the investigation of coherent acoustic phonons in semiconductors and semiconductor heterostructures and their prospect for high-frequency optical modulators. Sec. 4 summarizes applications of ASOPS in the field of ultrasonics.

\section{HIGH-SPEED ASYNCHRONOUS OPTICAL SAMPLING}

Experiments in the field of ultrafast time-domain spectroscopy generally require expertise from a number of different fields. Inherent difficulties of mostly technical nature have often limited the performance of femtosecond pump-probe techniques for practical applications such as electro-optic sampling of high speed circuits [13,14], laser based ultrasonics [10,15-18], for spintronics [19-21] or time-domain THz spectroscopy [22-24]. In addition, imaging applications in biology or materials research call for a minimization of the time required for the acquisition of a pump-probe trace in order to reduce the pixel dwell time. In this context, high-speed ASOPS may very well contribute to new spectroscopy and imaging applications since it provides $\mathrm{kHz}$ scan rates over a nanosecond time-delay window without the difficulties usually inherent to mechanical delay stages.

ASOPS provides the functionality of an all-optical oscilloscope at several $\mathrm{kHz}$ scan rate and eliminates the disadvantages of conventional pump-probe techniques due to the use of mechanical time-delay scanners. Conventional pump-probe techniques most commonly use trains of picosecond or femtosecond pulses derived from a single mode-locked laser such as femtosecond mode-locked Ti:sapphire solid-state lasers or Er-doped fiber lasers. Pump- and probe pulse trains are obtained via a beam splitter. One pulse train travels a variable distance and thus has a variable time-delay versus the other at the sample position. Mostly, time-delay scanning is accomplished by a retro-reflector mounted onto a motorized translation stage or a shaker-type device. Translation stages are capable of realizing several hundreds of picoseconds of time-delay and reach scanning speeds typically in the range of 10 ps per second depending on the signal averaging time per data point. Shaker-type devices like galvano-scanners or vibrating membranes have higher scanning frequencies in the range of $10 \mathrm{~Hz}$ to $100 \mathrm{~Hz}$, however, the maximum time-delay achievable is limited to approximately $100 \mathrm{ps}$ to $10 \mathrm{ps}$, respectively, depending on the mechanical resonance of the shaker device. For example, we have frequently employed a shaker operating in the range of $50 \mathrm{~Hz}$ to $100 \mathrm{~Hz}$ providing a time-delay of $10 \mathrm{ps}$ to $20 \mathrm{ps}$ [25-27]. Aside from the limited scan rate, a severe disadvantage of mechanical delay scanners is the fact that it is practically impossible to eliminate variations of the beam position and the beam diameter on the sample. These effects are due to residual pitch and yaw of translation stages as they travel several tens of centimeters to accomplish hundreds of picoseconds of timedelay as well as to the inherent beam divergence in the setup. In case of all optical pump-probe experiments these effects may cause artifacts that are impossible to distinguish from real sample dynamics.

ASOPS is based on two mode-locked lasers operating at repetition rates $f_{R I}$ and $f_{R 2}$, respectively. The repetition rates are linked at a fixed difference $\Delta f_{R}=f_{R 2}-f_{R I}$ in the range of $10 \mathrm{kHz}$. One of the lasers is used as pump laser to excite the sample, the second as probe laser to detect the optically induced transient response. The difference in the repetition rates of the two lasers results in a linear scanning of the time-delay between the two laser pulse trains with the frequency $\Delta f_{R}$. The time delay between pairs of pulses in the two pulse trains $\tau$ is given as $\tau(t)=\left(\Delta f_{R} / f_{R 2}\right) t \bmod \left(1 / f_{R 2}\right)$, with $t$ the real time and $f_{R 2}$ the repetition rate of the pump laser. The accessible time-delay window is given by the inverse repetition rate of the pump laser. The time-delaying scheme of ASOPS is illustrated in Fig. 1. Historically, ASOPS has been introduced first with actively mode-locked picosecond dye lasers at repetition rates around $100 \mathrm{MHz}$ [28]. A similar scheme employs two $50 \mathrm{MHz}$ femtosecond fiber lasers with modulated repetition rate difference to perform pump-probe spectroscopy without mechanical delay scanner [29]. The time-delay resolution of an ASOPS experiment, i.e. the 
frequency bandwidth of the all-optical oscilloscope, has two limitations. Firstly, the bandwidth $\mathrm{B}$ of the detection and data acquisition hardware limit the time-delay resolution $\Delta \tau$ to $\Delta \tau=\Delta \mathrm{f}_{\mathrm{R}} /\left(\mathrm{f}_{\mathrm{R} 2} \times \mathrm{B}\right)$. Typically, high-resolution $(\geq 14 \mathrm{Bit}) \mathrm{A} / \mathrm{D}$ converters reach values around $\mathrm{B}=100 \mathrm{MHz}$. At $\Delta \mathrm{f}_{\mathrm{R}}=10 \mathrm{kHz}$ and $\mathrm{f}_{\mathrm{R} 2}=1 \mathrm{GHz}$ a time-delay resolution $\Delta \tau$ of $100 \mathrm{fs}$ results which is sufficient for spectroscopy into the multiple $\mathrm{THz}$ frequency range. Secondly, residual timing jitter of the femtosecond lasers versus their reference oscillator and timing jitter between the reference oscillators cause an instability of the time-base $\tau(\mathrm{t})$, resulting in another limitation to $\Delta \tau$. Generally, this effect becomes disproportionately more pronounced as $\Delta \mathrm{f}_{\mathrm{R}}$ is reduced much below $10 \mathrm{kHz}$ because the time between two successive scans is increased thus providing more time for residual timing jitter to accumulate before the next trigger event occurs. The above discussed effects make it practically impossible to use femtosecond lasers with repetition rates much below $1 \mathrm{GHz}$ and/or scan rates $\Delta \mathrm{f}_{\mathrm{R}}$ much below $10 \mathrm{kHz}$.

Our high-speed implementation of ASOPS is based on two modelocked Ti:sapphire femtosecond lasers at $\sim 1 \mathrm{GHz}$ repetition rate [30]. Our setup thus covers a temporal measurement window of $\sim 1$ ns that we choose to scan at $\Delta \mathrm{f}_{R}=10$ $\mathrm{kHz}$. The effective bandwidth of our detection and data acquisition hardware is $\mathrm{B}=100 \mathrm{MHz}$ thus limiting the time-delay resolution to $100 \mathrm{fs}$. However, the practical limitation to the time-resolution is given by the active repetition rate stabilization. For the present setup this will be discussed later. The first realization of high-speed ASOPS with GHz repetition rates has been implemented for laser-based ultrasonics on silicon-on-insulator for semiconductor metrology applications [31]. However, at that time the stabilization electronics for the repetition rates was not optimized, hence a time resolution in the order of $10 \mathrm{ps}$ was achieved. Later, high-speed ASOPS has been employed for time-resolved THz spectroscopy with a time-resolution of $\sim 2.5 \mathrm{ps}$ corresponding to a frequency bandwidth up to $400 \mathrm{GHz}$ [32]. Subsequently, extensive transfer of knowledge about laser stabilization techniques for $1 \mathrm{GHz}$ femtosecond lasers from the field of ultrastable frequency combs has brought about significant improvements for the time-delay resolution of high-speed ASOPS [33-35]. By employing advanced stabilization electronics, we recently improved the time-resolution of high-speed ASOPS into the range of $200 \mathrm{fs}$, providing a bandwidth of up to $5 \mathrm{THz}[4,36]$. Current performance is not longer limited by the stabilization electronics but by the employed reference oscillators.

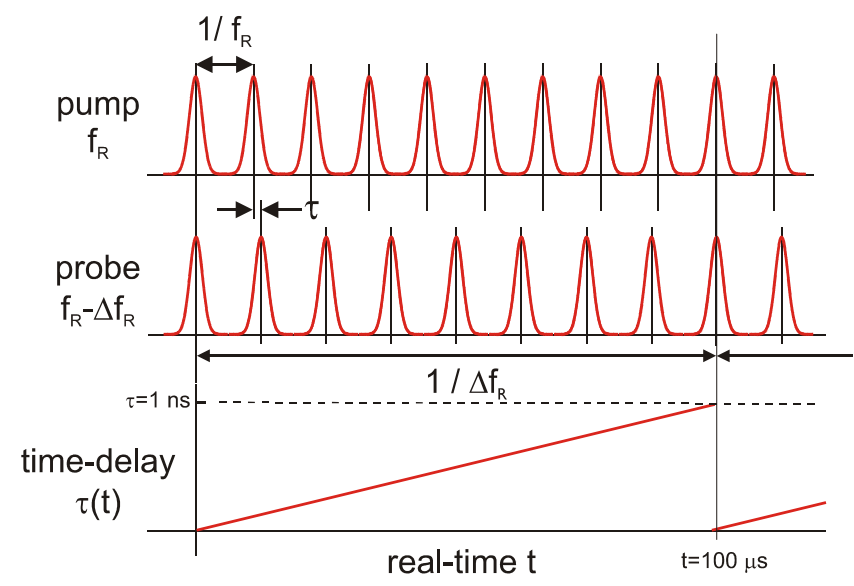

Fig. 1: Schematic sketch of the ASOPS time-delaying principle with two femtosecond lasers at approximately $1 \mathrm{GHz}$ repetition rate $\left(f_{R} \sim 1 \mathrm{GHz}\right)$ and a repetition rate difference $\Delta \mathrm{f}_{\mathrm{R}}=10 \mathrm{kHz}$. 


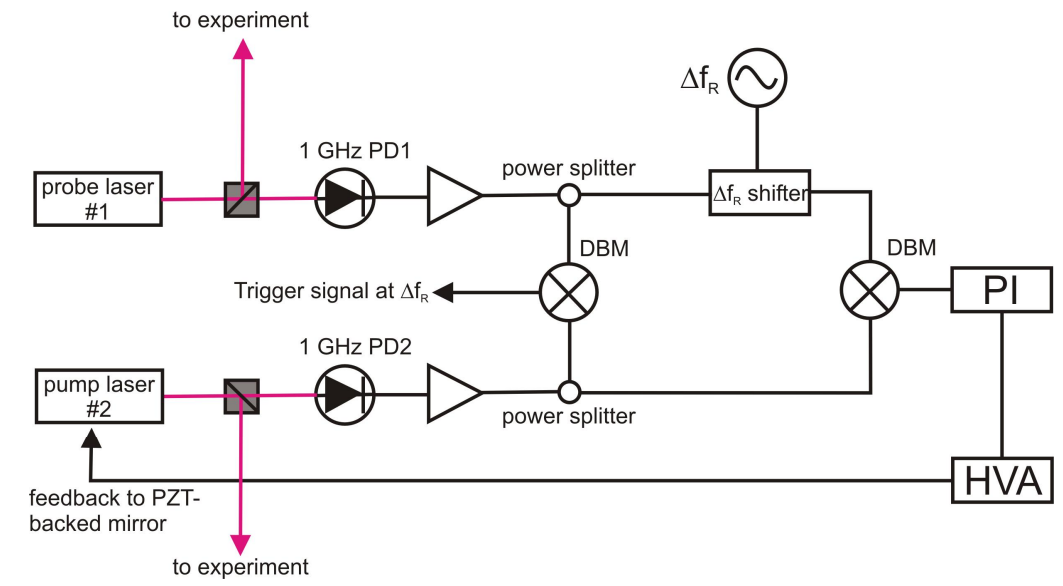

Fig. 2: Schematic sketch of the ASOPS pump-probe set-up. PD1 and PD2 are photodiodes, DBM is a double-balanced mixer. PI is an adjustable proportional-integral loop amplifier and HVA is a high-voltage amplifier.

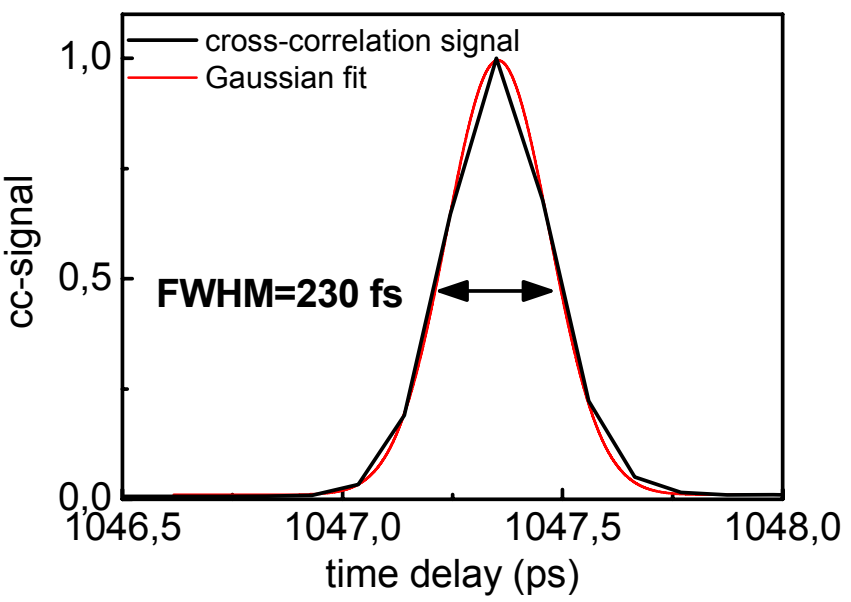

Fig. 3: Cross-correlation signal of the two femstosecond lasers averaged over 100,000 single scans traces over $\sim 10 \mathrm{~s}$ of data acquisition time. Signal has been chosen to appear approximately at the end of one full accessible measurement window (in the case of this measurement $\mathrm{f}_{\mathrm{R} 2}{ }^{-1}=(950 \mathrm{MHz})^{-1}=1053 \mathrm{ps}$ ) after the trigger event where timing jitter is expected to be largest.

In the present work, two stabilization schemes have been employed: In one possible scheme, both lasers are stabilized to individual synthesizers (models SML01 from Rohde\&Schwarz) that share a common $10 \mathrm{MHz}$ reference oscillator and the difference frequency $\Delta f_{R}$ is set with the synthesizers. This scheme is limited by phase-noise intrinsic to the synthesis process of each synthesizer. At $\Delta \mathrm{f}_{\mathrm{R}}=10 \mathrm{kHz}$ a time-delay resolution of $280 \mathrm{fs}$ has been obtained. If $\Delta \mathrm{f}_{\mathrm{R}}$ is reduced to 1 $\mathrm{kHz}$, lower frequency phase-noise contributions of the synthesizers increasingly compromise the system and result in a time-delay resolution of worse than $1 \mathrm{ps}$. Higher scan-rates $\Delta \mathrm{f}_{\mathrm{R}}$ are impractical due to bandwidth limitations given by the detection and data acquisition hardware $\mathrm{B}$. In a second scheme, the repetition rate $f_{R I}$ of the probe laser is detected with a fast photodiode (PD1) and up-shifted to the frequency $f_{R 2}=f_{R 1}+\Delta f_{R}$ by $10 \mathrm{kHz}$ using a frequency-shifter. The frequencyshifter consists of a single-sideband generator with an additional carrier suppression stage that suppresses the initial carrier-signal and mirror frequencies by better than $50 \mathrm{~dB}$. The frequency of laser $2, f_{R 2}$, is detected with a second photodiode (PD2) and phase-locked to the frequency-shifted repetition rate signal of the probe laser. We use a doublebalanced mixer (DBM) as phase-detector and feed its output back to a piezoelectric transducer that controls the position of a cavity mirror in laser 2 via an adjustable loop-amplifier. In order to characterize the jitter of the time-base $\tau(t)$ we 
detect a cross-correlation signal between the two lasers using non-collinear sum-frequency generation in a $\beta$-bariumborate-crystal. The cross-correlation signal is detected with a $125-\mathrm{MHz}$ bandwidth photoreceiver and recorded using a 14-Bit $100 \mathrm{MHz}$ digitizer board. An electronic trigger signal at $\Delta f_{R}$ for the digitizer is generated via a second DBM that has the signals from PD1 and PD2 as inputs. The real-time scale is converted to a time-delay scale $\tau(t)$ through the factor $\Delta f_{R} / f_{R 2}$. Fig. 3 shows an average over 100,000 measurements acquired over approx. $10 \mathrm{~s}$ where we have chosen the cross-correlation signal to appear after approximately one full measurement window after the trigger event. In such a configuration, the $230 \mathrm{fs}$ width of the signal gives an upper limit for the time-base jitter at all earlier times within the window. The pulse duration emitted from each laser is approx. $50 \mathrm{fs}$. The current limit to the time resolution are residual contributions at the carrier frequency at the output of the frequency shifter that cause unwanted modulations of the error signal that controls the pump laser repetition rate. An improvement of the timing stability to the sub-100 fs regime is possible by performing the phase-lock at a higher harmonic of the repetition rate or by using digital phase-locking techniques.

\section{COHERENT ACOUSTIC PHONON DYNAMICS IN SUPERLATTICES}

The acoustic phonon spectrum in a superlattice is determined by the back-folding of the bulk phonon dispersion into the mini-Brillouin zone of the periodic heterostructures. The dispersion relation of the superlattice zone-folded modes can be calculated on the basis of a layered elastic continuum model, known as the Rytov model [37]. Zone-folding of the phonon dispersion results in a series of acoustic phonon branches with finite frequencies at vanishing wavevectors. The frequency of the modes at zero wavevector is determined by the sound velocities and the thickness of the constituting layers, repsectively. Fig. 4 depicts the acoustic phonon of a GaAs-AlAs superlattice consisting of 19 monolayers GaAs and 19 monolayers AlAs, referred to as (19/19) superlattice, as calculated from the Rytov model. The frequency of the first back-folded branch at zero wavevector is approx. $480 \mathrm{GHz}$. This frequency can be tailored through the parameters of the superlattice. Zone-folded acoustic phonons in semiconductor superlattice have been investigated through $\mathrm{CW}$ Raman scattering experiments [5,6]. The coherent excitation of coherent acoustic phonons in the THz frequency range by femtosecond laser pulses was first observed by Yamamoto et al. in GaAs/AlAs superlattices [38]. The identification of impulsive stimulated Raman scattering (ISRS) as the relevant excitation mechanism of coherent LA phonon in GaAs/AlAs superlattice was given later [39,40]. Recently, the coherent excitation of TA phonons was observed in superlattices grown on low-symmetry planes, e.g. on [311] and [211] orientation [41].

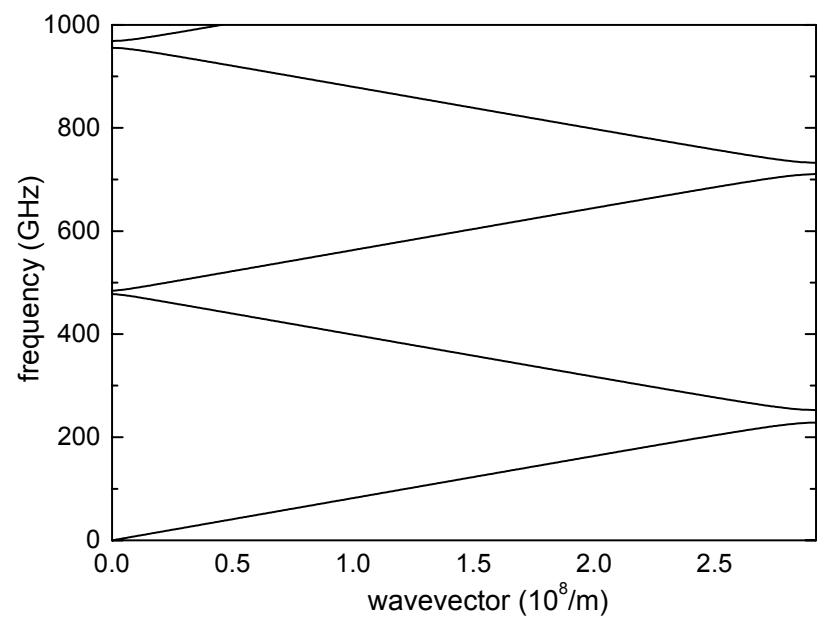

Fig. 4: Dispersion relation of acoustic zone-folded modes in a (19/19) GaAs-AlAs superlattice. 
Figure 5 depicts the reflectivity changes recorded with the ASOPS technique at room-temperature from the $(19 / 19)$ GaAs-AlAs superlattice consisting of 40 periods. The pump and the probe lasers were set to the first electron-hole interband resonance of the superlattice at $800 \mathrm{~nm}$. Fig. 5 (a) shows the changes of the reflectivity induced by the impulsive generation of electron-hole pairs in the superlattice. The reflectivity changes decay through the recombination and diffusion of the carriers and are of the order of $10^{-4} \Delta \mathrm{R} / \mathrm{R}_{0}$. These reflectivity changes are modulated by coherent zone-folded LA phonons with an amplitude of the order of $10^{-6} \Delta \mathrm{R} / \mathrm{R}_{0}$. The coherent phonon signature is extracted from the data by subtracting the electronic background as an exponential decay (Fig. 5 b). The Fourier spectrum of the phonons is shown in Fig. 5 c). The Fourier spectrum reveals a strong modulation through a mode close to $40 \mathrm{GHz}$, which is the Brillouin branch of the phonon spectrum (see Fig. 4). Additionally the first and second order back-folded modes are clearly resolved in the spectrum at approx. $480 \mathrm{GHz}$ and $960 \mathrm{GHz}$, respectively. These frequencies correspond well with the calculated phonon dispersion shown in Fig. 4. They appear as triplets, since the detection process selects the modes at zero wavevector and those at twice the wavevector of the probe light [39].
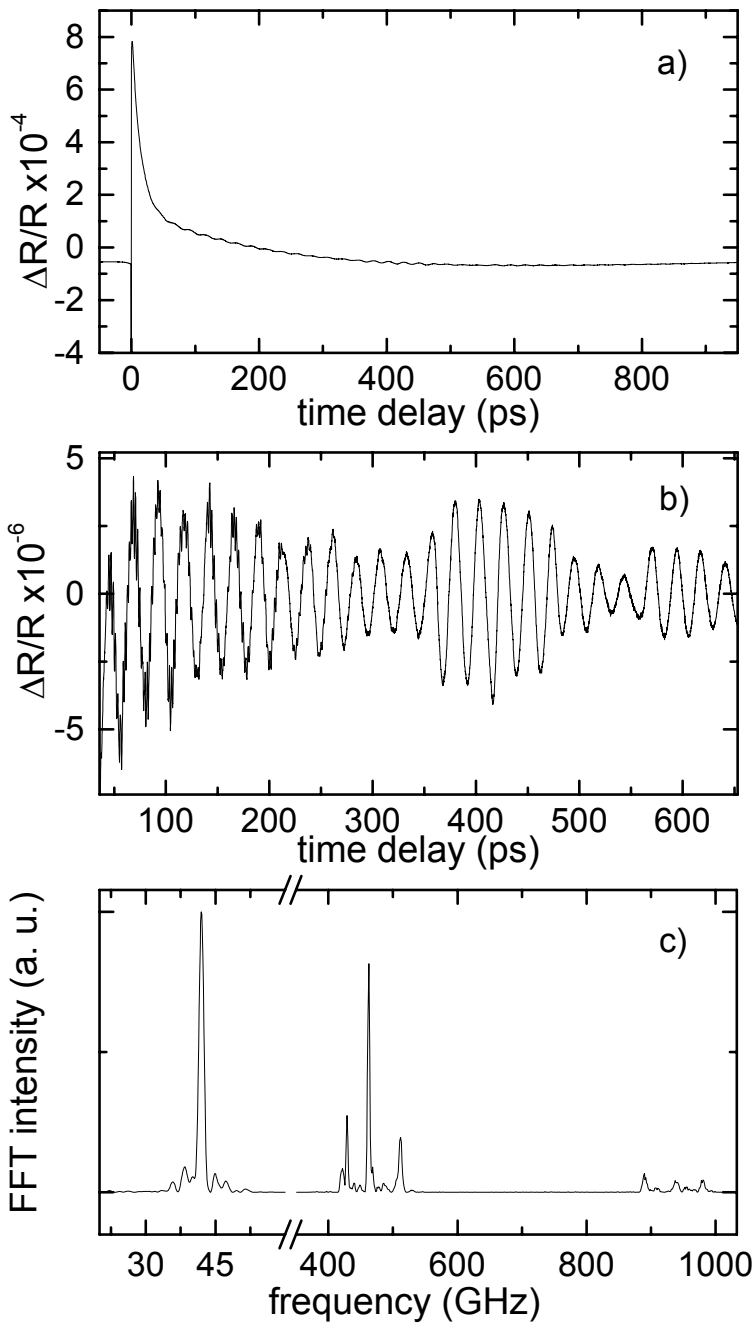

Fig. 5: (a) Time-resolved reflectivity changes of a (19/19) GaAs-AlAs superlattice recorded over 1 nanosecond. (b) Extracted coherent acoustic phonon contribution to the signal in (a) shown for 800 ps. (c) Numerical Fourier transform of the signal in (b). 
Recently, superlattices embedded in microcavities have been proposed for a strong enhancement of the interaction of acoustic phonons with light. A resonator for acoustic phonons can be constructed in the same way a microcavity is constructed for photons. The resonance wavelength of the acoustic mode has to be chosen to lie within one of the minigaps of the zone-folded acoustic phonon dispersion. In a coupled phonon-photon cavity designed this way an enhancement in the Raman scattered intensity of five orders of magnitude has been observed $[7,8]$. These ideas open the pathway to design semiconductor heterostructures for modulating light at frequencies in the $\mathrm{GHz}$ to $\mathrm{THz}$ range through acoustic phonons similar to well known acousto-optic modulators. The enhancement of the sound-light interaction in heterostructures has not yet been demonstrated in experiments in the time-domain.

In order to prove the principle of the manipulation of coherent acoustic phonons via optical pulses we use the ASOPS technique and send the pump-pulse through an optical pulse-shaper consisting of two liquid crystal modulators for modulating the amplitude and phase of the femtosecond pulses [3]. The coherent control of acoustic phonons in superlattices has been demonstrated previously through two pulses derived from a beam splitter and a delay stage in the pump beam path [42]. Fig. 6 (a) shows the normalized changes of the reflectivity for two excitation conditions of one and two excitation pulses. Both pulses lead to the excitation of electron-hole pairs in the superlattice. The pulse distance is chosen to equal half the period of the first order back-folded phonon modes at approx. $460 \mathrm{GHz}$, i.e. 1.1 ps. The average excitation power of the single pulse is $190 \mathrm{~mW}$ and that for the two pulses is $120 \mathrm{~mW}$. Clearly, the destructive interference of the coherent phonons generated in the two-pulse experiment is demonstrated.

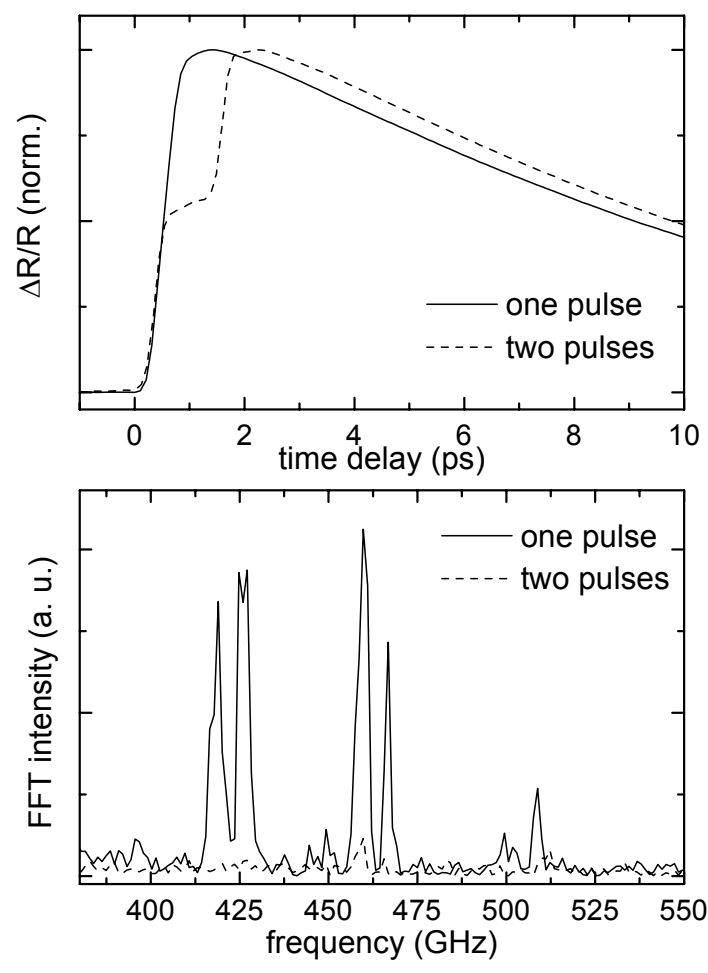

Fig. 6: Demonstration of coherent control of the amplitude of coherently generated acoustic phonons in the $\mathrm{THz}$ frequency range via two-pulse excitation. Upper figure: Reflectivity change in the first $10 \mathrm{ps}$ after excitation with a single pulse (solid line) and double-pulses (dashed line). Lower figure: Fourier transform of coherently excited acoustic phonons in the range of $400 \mathrm{GHz}$ for single pulse (solid line) and double pulse excitation (dashed line). 


\section{APPLICATIONS OF ASOPS FOR LASER BASED ULTRASONICS IN SEMICONDUCTOR METROLOGY}

Acoustic pulses generated through pulsed laser excitation have been used in the past to study phonon propagation in layered structures. The transit times of acoustic pulses allows the determination of layer thicknesses in a non-invasive way. This technique and its applications are referred to as laser based ultrasonics [10,11]. Using femtosecond pulses in conjunction with appropriate models for the acoustic pulse propagation a depth resolution in the sub-nm can be obtained making this technique appropriate for semiconductor metrology [43]. The typical time-scales for the detection of acoustic pulse propagation in multilayers is in the $100 \mathrm{ps}$ range. Taking a Si layer of $1 \mu \mathrm{m}$ thickness gives a transit time of an acoustic pulse through this layer of 118 ps. For this time scale the ASOPS technique is ideally suited as we demonstrate in two application examples.

Fig. 7 (inset) shows the time resolved reflectivity changes obtained from a $324 \mathrm{~nm} \mathrm{Si}$ layer wafer bonded onto a $\mathrm{CoSi}_{2}$ layer. The whole structure of a silicon-on-metal-on-insulator consists of a top $\mathrm{Si}$ layer, a buried $\mathrm{CoSi}_{2}$ layer and a buried $\mathrm{SiO}_{2}$ layer on a $\mathrm{Si}(100)$ substrate which is formed using Co silicidation, wafer bonding and wafer splitting. These substrates are employed in a BiCMOS process [44]. The reflectivity change of this substrate is modulated with equally spaced acoustic pulses from multiple reflections of the acoustic pulse. The roundtrip time of these pulses running back and forth through the top Si layer is $77 \mathrm{ps}$. Taking a longitudinal sound velocity of $8.43 \times 10^{5} \mathrm{~cm} / \mathrm{s}$ for the [001] direction of silicon, this corresponds to a layer thickness of $324 \mathrm{~nm}$ in good agreement with TEM results on this sample.

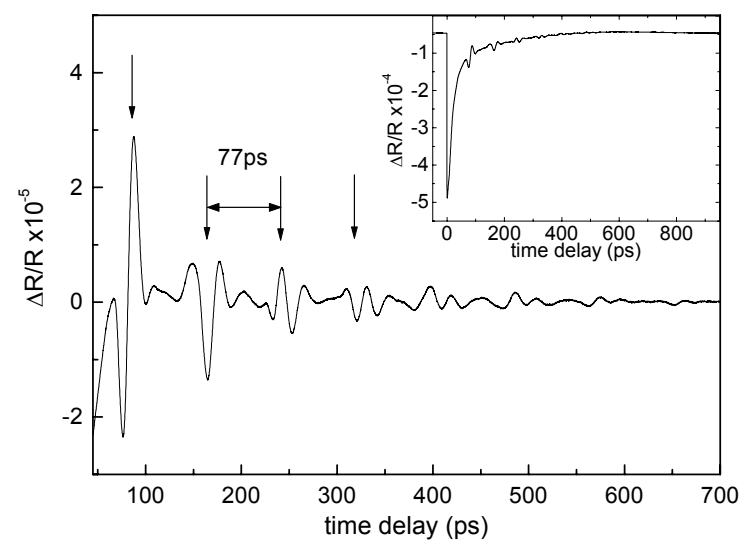

Fig. 7: (a) Time-resolved reflectivity changes from a $324 \mathrm{~nm}$ thick Si layer wafer-bonded on $\mathrm{CoSi}_{2}$. The arrows mark the time delays for which reflections of the acoustic pulse appear at the surface.

The second application demonstration is the investigation of Si-Mo multilayers which have been fabricated as Bragg mirrors for EUV radiation in the $12 \mathrm{~nm}-15 \mathrm{~nm}$ wavelength range [45,46]. Such multi-layers have been previously investigated with pump-probe spectroscopy based on a conventional system employing a mechanical delay line [47,48]. The time-resolved reflectivity changes from a structure consisting of 50 periods with a period of $6.84 \mathrm{~nm}$ over the first $150 \mathrm{ps}$. The ratio of the thickness of the Mo layer to the total period is 0.38 . The reflectivity change shows an electronic relaxation which is modulated in the first 20 ps by coherent phonons of the multilayer. After 110 ps an acoustic echo is observed which corresponds to the roundtrip time of an acoustic pulse running from the surface to the substrate and back. In Fig. 8 (b) the extracted phonon signature at early time delays is shown. The Fourier transform (Fig. 8 c)) reveals a pronounced maximum at $1.12 \mathrm{THz}$ and a minimum at $1.02 \mathrm{THz}$. A second smaller feature is observed at $0.4 \mathrm{THz}$. All features correspond very well with a theoretical calculation of the surface modes of this structure $[47,48]$. The specific details of this spectrum allow the non-invasive characterization of the composition of the EUV mirrors such as the ratio of the $\mathrm{Mo} / \mathrm{Si}$ thickness and the interface quality. 

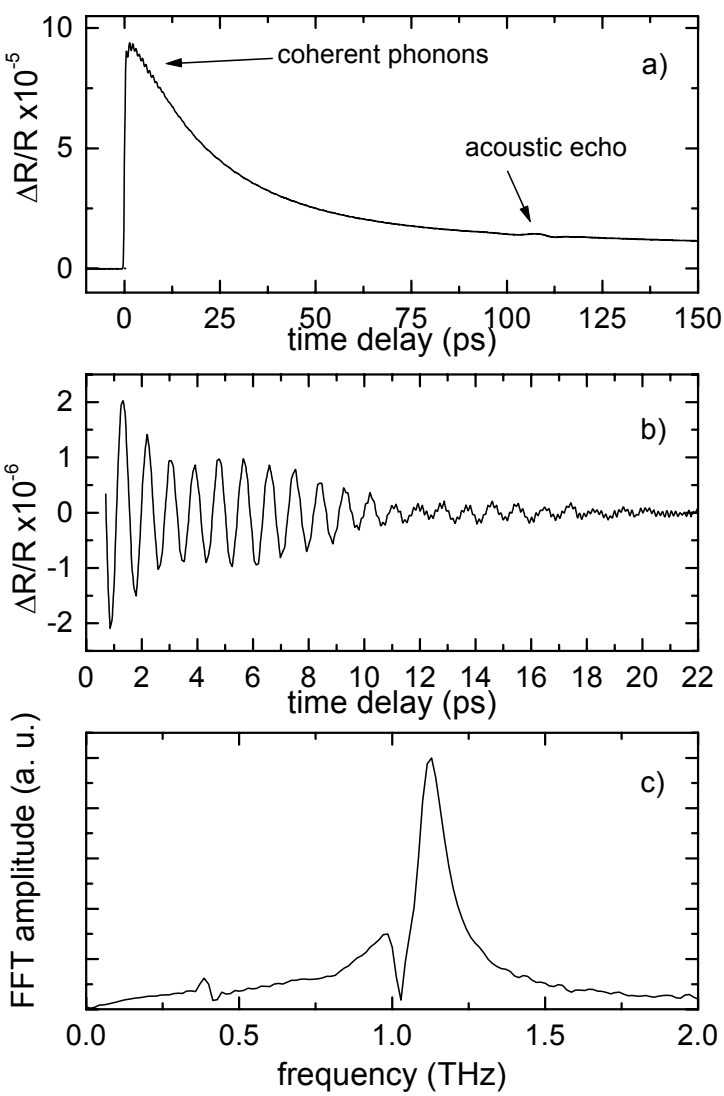

Fig. 8: (a) Time-resolved reflectivity change of a Si/Mo EUV mirror. (b) Extracted coherent phonon oscillations in the first 20 ps. (c) Numerical Fourier transform of the data in (b).

\section{CONCLUSIONS}

We have presented a new optical pump-probe technique which provides $100 \mathrm{fs}$ time resolution at scan rates of $10 \mathrm{kHz}$ over a nanosecond time-delay. This implementation of asynchronous optical sampling (ASOPS) exploits the advantages of using two femtosecond lasers with $\mathrm{GHz}$ repetition rates and - in conjunction with a A/D converter board - has the functionality of an all-optical oscilloscope. The dynamics of coherent acoustic phonons in the $\mathrm{GHz}$ to $\mathrm{THz}$ frequency range are recorded with high-resolution and high-sensitivity. Coherent acoustic phonons in semiconductor superlattices as well as acoustic pulse propagation in nanoscale multi-layers of relevance for microelectronic applications have been observed. The application of coherent acoustic for the high-frequency modulation of optoelectronic signals in combined photon-phonon cavities has been discussed.

\section{ACKNOWLEDGMENTS}

We like to thank A.J. Kent. A. Fainstein, J. Vollmann, and N.W. Pu for stimulating discussions. We acknowledge the financial support through the Deutsche Forschungsgemeinschaft (DE456/9) and the Ministry of Science, Research and the Arts of Baden-Württemberg 


\section{REFERENCES}

1. S. Shah, Ultrafast Spectroscopy of Semiconductors and Semiconductor Nanostructures, $2^{\text {nd }}$ edition, Springer Berlin (1999).

2. T. Dekorsy, G. Cho, H. Kurz, "Coherent phonons in condensed media", in Light Scattering in Solids VIII, Topics in Appl. Phys.(76), eds M. Cardona and G. Güntherodt, Springer, Berlin (2000) pp. 169-209.

3. A.M. Weiner, "Femtosecond pulse shaping using spatial light modulators", Rev. Sc. Instr. 71, 1929 (2000).

4. A. Bartels, F. Hudert, C. Janke, T. Dekorsy, and K. Köhler, "Femtosecond time-resolved optical pump-probe spectroscopy at kHz-scan-rates over ns-time-delays without mechanical delay line", Appl. Phys. Lett. 88, 041117 (2006).

5. C. Colvard, R. Merlin, M. V. Klein, and A. C. Gossard, "Observation of folded acoustic phonons in a semiconductor superlattice", Phys. Rev. Lett. 45, 298 (1980).

6. C. Colvard, T. A. Gant, M. V. Klein, R. Merlin, R. Fischer, H. Morkoc, and A. C. Gossard, "Folded acoustic and quantized optic phonons in (GaAl)As superlattices", Phys. Rev. B 31, 2080 (1985).

7. M. Trigo, A. Bruchhausen, A. Fainstein, B. Jusserand, and V. Thierry-Mieg, "Confinement of Acoustical Vibrations in a Semiconductor Planar Phonon Cavity", Phys. Rev. Lett. 89, 227402 (2002).

8. P. Lacharmoise, A. Fainstein, B. Jusserand, and V. Thierry-Mieg, "Optical cavity enhancement of light-sound interaction in acoustic phonon cavities ", Appl. Phys. Lett. 84, 3274 (2004).

9. N. D. Lanzillotti-Kimura, A. Fainstein, A. Lemaître, and B. Jusserand, "Nanowave devices for terahertz acoustic phonons", Appl. Phys. Lett. 88, 083113 (2006).

10. H. Marris, "Picosecond ultrasonics", Sc. American 1, 64 (1998).

11. H.Y. Hao, H.J. Maris and D.K. Sadana, "Non-destructive evaluation of interfaces in bonded silicon-on-insulator structures using the picosecond ultrasonics technique", Electrochemical and Solid State Letters 54, 1 (1998).

12. B.C. Daly, J. Klein, T.B. Norris, S. Pau, D.M. Tennant, J.A. Taylor, J.E Bower, "Nanoscale coherent acoustic phonon imaging ", Proceedings of SPIE 6118, art. no. 61180U (2006).

13. T. Pfeifer, H.M. Heiliger, T. Löffler, C. Ohlhoff, C. Meyer, G. Lüpke, H.G. Roskos, and H. Kurz, "Optoelectronic on-chip characterization of ultrafast electric devices: Measurement techniques and applications ", IEEE J. Sel. Top. Quantum Electr. 2, 586 (1996).

14. M. Bieler, M. Spitzer, G. Hein, U. Siegner, and E.O Göbel, "Optoelectronic measurement of the transfer function and time response of a $70 \mathrm{GHz}$ sampling oscilloscope ", Appl. Phys. A 78, 429 (2004).

15. C. Thomsen, H.T. Grahn, H.J. Maris, and J. Tauc, "Surface generation and detection of phonons by picosecond light pulses", Phys. Rev. B 34, 4129 (1986).

16. O.L. Muskens and J.I. Dijkhuis, "Inelastic light scattering by trains of ultrashort acoustic solitons in sapphire", Phys. Rev. B 70, 104301 (2004).

17. G.D. Cahill, W.K. Ford, K.E. Goodson, G.D. Mahan, A. Majumdar, H.J. Maris, R. Merlin, and S.R. Phillpot, "Nanoscale thermal transport", J. Appl. Phys. 93, 793 (2003).

18. O. Matsuda, T. Tachizaki, T. Fukui, J.J. Baumberg, O.B. Wright, "Acoustic phonon generation and detection in GaAs/AlGaAs quantum wells with picosecond laser pulses", Phys. Rev. B 71 (11), 115330 (2005)

19. J.M. Kikkawa and D.D. Awschalom, "Resonant Spin Amplification in $n$-Type GaAs", Phys. Rev. Lett. 80, 4313 (1998).

20. J.M. Kikkawa and D.D. Awschalom, "All-Optical Magnetic Resonance in Semiconductors", Science 287, 473 (2000).

21. R.C. Myers, K.C. Ku, X. Li, M. Samarth, and D.D. Awschalom, "Optoelectronic control of spin dynamics at nearterahertz frequencies in magnetically doped quantum wells", Phys. Rev. B 72, 041302 (2005).

22. D. Mittleman (ed.), Sensing with Terahertz Radiation, Springer, Berlin (2004).

23. K. Sakai (ed.), Terahertz Optoelectronics, Springer, Berlin (2005).

24. R.A. Cheville and D.Grischkowsky, IEEE Trans. Microwave Theory Tech. 38, 1684 (1995).

25. T. Dekorsy, A. Bartels, H. Kurz, K. Köhler, R. Hey, and K. Ploog, "Coupled Bloch-phonon oscillations in semiconductor superlattices", Phys. Rev. Lett. 85, 1080 (2000).

26. A. Bartels, T. Dekorsy, and H. Kurz, "Impulsive excitation of phonon-pair-combination states by second-order Raman scattering in KTaO3”, Phys. Rev. Lett. 84, 2981 (2000). 
27. G.C. Cho, H.J. Bakker, T. Dekorsy, and H. Kurz, "Time-resolved observation of coherent phonons by the FranzKeldysh effect", Phys. Rev. B. 53, 6904 (1996).

28. P.A. Elzinga, F.E. Lytle, Y. Jian, G.B. King, and N.M. Laurendeau, "Pump/probe method for fast analysis of visible spectral signatures utilizing asynchronous optical sampling", Appl. Opt. 26, 4303 (1987).

29. G. Sucha, M.E. Fermann, D.J. Harter, and M. Hofer "A new method for rapid temporal scanning of ultrafast lasers", IEEE Journal on Selected Topics in Quantum Electronics 2, 605 (1996).

30. A. Bartels, T. Dekorsy, and H. Kurz, "Unidirectionally modelocked fs ring laser at $2 \mathrm{GHz}$ repetition rate and its applications in spectroscopy", Opt. Lett. 24, 996 (1999).

31. A. Bartels, Ph.D. Thesis, RWTH Aachen (2000).

32. C. Janke, M. Först, M. Nagel, H. Kurz, and A. Bartels, "Asynchronous optical sampling for high-speed characterization of integrated resonant terahertz sensors", Opt. Lett. 30, 1405 (2005).

33. A. Bartels, S.A. Diddams, T.M. Ramond, and L. Hollberg, "Mode-locked laser pulse trains with subfemtosecond timing jitter synchronized to an optical reference oscillator", Opt. Lett. 28, 663 (2003).

34. A. Bartels, C.W. Oates, L. Hollberg, and S.A. Diddams, "Stabilization of femtosecond laser frequency combs with subhertz residual linewidths", Opt. Lett. 29, 1081 (2004).

35. A. Bartels, S.A. Diddams, C.W. Oates, G. Wilpers, J.C. Bergquist, W.H. Oskay, and L. Hollberg, "Femtosecond laser based synthesis of ultrastable microwave signals from optical frequency references", Opt. Lett. 30, 667 (2005).

36. A. Bartels, A. Thoma, C. Janke, T. Dekorsy, A. Dreyhaupt, S. Winnerl, and M. Helm, "High-resolution THz spectrometer with kHz scan rates", Opt. Expr. 14, 430 (2006).

37. S. M. Rytov, Akust. Zh. 2, 71 (1956) [Sov. Phys. Acoust. 2, 68 (1956)].

38. A. Yamamoto, T. Mishina, Y. Masumoto, M. Nakayama, "Coherent oscillation of zone-folded phonon modes in GaAs-AlAs superlattices", Phys. Rev. Lett. 73, 740 (1994).

39. K. Mizoguchi, M. Hase, S. Nakashima, and M. Nakayama, "Observation of coherent folded acoustic phonons propagating in a GaAs/AlAs superlattice by two-color pump-probe spectroscopy", Phys. Rev. B 60, 8262 (1999).

40. A. Bartels, T. Dekorsy, H. Kurz, and K. Köhler, "Coherent Zone-Folded Longitudinal Acoustic Phonons in Semiconductor Superlattices: Excitation and Detection", Phys. Rev. Lett. 82, 1044 (1999)

41. R. N. Kini, A. J. Kent, N. M. Stanton, and M. Henini, "Generation and detection of terahertz coherent transversepolarized acoustic phonons by ultrafast optical excitation of GaAs/AlAs superlattices", Appl. Phys. Lett. 88, $134112(2006)$

42. A. Bartels, T. Dekorsy, H. Kurz, and K. Köhler, "Coherent control of acoustic phonons in semiconductor superlattices", Appl. Phys. Lett. 72, 2844 (1998)

43. http://www.rudolphtech.com/pdfs/products/PULSE_Technology.pdf

44. Q.T.Zhao, H. L. Bay, S. Zimmermann, M. Wiemer, C. Kaufmann, B. Trui, H. Höhnemann, V. Dudek, and S. Mantl, "Thermal stability of $\mathrm{CoSi}_{2}$ layers implemented in a silicon-on-insulator substrate", Semicond. Sci. Technol. 21, 157-161 (2006).

45. S. Braun, H. Mai, M. Moss, R. Scholz, and A. Leson, "Mo/Si multilayers with different barrier layers for applications as EUV mirrors", Jap. J. Appl. Phys. 414074 (2002).

46. S. Braun, T. Foltyn, L. Van Loyen, M. Moss, A. Leson, "Multi-component EUV multilayer mirrors", Proceedings of SPIE - 5037 I, pp. 274-285 (2003).

47. N.W. Pu and J. Bokor, "Study of surface and bulk acoustic phonon excitations in superlattices", Phys. Rev. Lett. 91, 076101 (2003).

48. N.W. Pu, "Ultrafast excitation and detection of acoustic phonon modes in superlattices", Phys. Rev. B 72, 115428 (2005). 